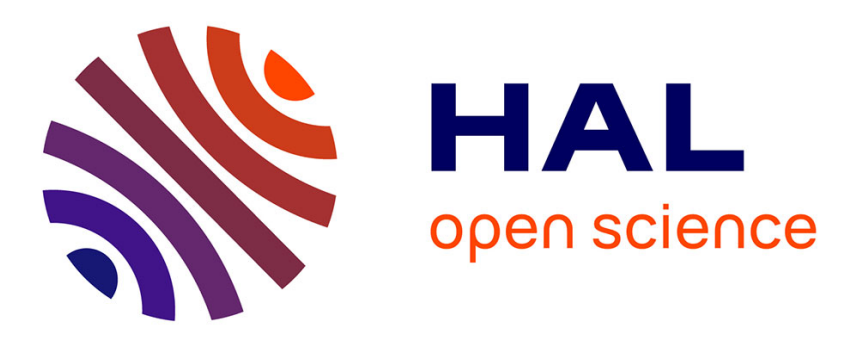

\title{
Electrical Tuning of the SERS Enhancement by Precise Defect Density Control
}

Canliang Zhou, Linfeng Sun, Fengquan Zhang, Chenjie Gu, Shuwen Zeng, Tao Jiang, Xiang Shen, Diing Shenp Ang, Jun Zhou

\section{- To cite this version:}

Canliang Zhou, Linfeng Sun, Fengquan Zhang, Chenjie Gu, Shuwen Zeng, et al.. Electrical Tuning of the SERS Enhancement by Precise Defect Density Control. ACS Applied Materials \& Interfaces, 2019, 11 (37), pp.34091-34099. 10.1021/acsami.9b10856 . hal-02927661

\section{HAL Id: hal-02927661 \\ https://hal-unilim.archives-ouvertes.fr/hal-02927661}

Submitted on 1 Sep 2020

HAL is a multi-disciplinary open access archive for the deposit and dissemination of scientific research documents, whether they are published or not. The documents may come from teaching and research institutions in France or abroad, or from public or private research centers.
L'archive ouverte pluridisciplinaire HAL, est destinée au dépôt et à la diffusion de documents scientifiques de niveau recherche, publiés ou non, émanant des établissements d'enseignement et de recherche français ou étrangers, des laboratoires publics ou privés. 


\section{ACS APPLIED MATERIALS \&INTERFACES}

\section{Functional Inorganic Materials and Devices}

Subscriber access provided by Columbia University Libraries

Electrical Tuning of the SERS Enhancement by Precise Defect Density Control

Canliang Zhou, Linfeng Sun, Fengquan Zhang, Chenjie Gu, Shuwen

Zeng, Tao Jiang, Xiang Shen, Diing Shenp Ang, and Jun Zhou

ACS Appl. Mater. Interfaces, Just Accepted Manuscript • DOI: 10.1021/acsami.9b10856 • Publication Date (Web): 21 Aug 2019

Downloaded from pubs.acs.org on August 26, 2019

\section{Just Accepted}

"Just Accepted" manuscripts have been peer-reviewed and accepted for publication. They are posted online prior to technical editing, formatting for publication and author proofing. The American Chemical Society provides "Just Accepted" as a service to the research community to expedite the dissemination of scientific material as soon as possible after acceptance. "Just Accepted" manuscripts appear in full in PDF format accompanied by an HTML abstract. "Just Accepted" manuscripts have been fully peer reviewed, but should not be considered the official version of record. They are citable by the Digital Object Identifier (DOI®). "Just Accepted" is an optional service offered to authors. Therefore, the "Just Accepted" Web site may not include all articles that will be published in the journal. After a manuscript is technically edited and formatted, it will be removed from the "Just Accepted" Web site and published as an ASAP article. Note that technical editing may introduce minor changes to the manuscript text and/or graphics which could affect content, and all legal disclaimers and ethical guidelines that apply to the journal pertain. ACS cannot be held responsible for errors or consequences arising from the use of information contained in these "Just Accepted" manuscripts. 


\title{
Electrical Tuning of the SERS Enhancement by
}

\section{Precise Defect Density Control}

\author{
Canliang Zhou ${ }^{\dagger}$, Linfeng Sun ${ }^{\star}$, Fengquan Zhang ${ }^{\dagger}$, Chenjie Gu*t, Shuwen Zeng*|, Tao Jiang ${ }^{\dagger}$, \\ Xiang Shen ${ }^{\#}$, Diing Shenp Ang ${ }^{\star}$ and Jun Zhou*广 \\ $\dagger$ Institute of Photonics, Ningbo University, 818 Feng Hua Rd. 315211, Ningbo, China \\ $\ddagger$ Department of Energy Science, Sungkyunkwan University, Suwon 16419, Korea \\ " XLIM Research Institute, UMR 7252 CNRS/University of Limoges, Avenue Albert Thomas \\ 87060, France
}

\# Research Institute of Advanced Technologies, Ningbo University, 818 Feng Hua Rd. 315211, Ningbo, China

\& School of Electrical and Electronic Engineering, Nanyang Technological University, 50

Nanyang Avenue 639798, Singapore

E-mail: guchenjie@nbu.edu.cn; zhoujun@nbu.edu.cn; shuwen.zeng@unilim.fr. 


\begin{abstract}
Surface-enhanced Raman scattering (SERS) has been widely established as a powerful analytical technique in molecular fingerprint recognition. Although conventional noble metal-based SERS substrates show admirable enhancement of the Raman signals, challenges on reproducibility, bio-compatibility and costs limit their implementations as the preferred analysis platforms. Recently, researches on SERS substrates have found that some innovatively prepared metal oxides/chalcogenides could produce noble metal comparable SERS enhancement, which profoundly expanded the material selection. Nevertheless, to tune the SERS enhancement of these materials, careful experimental designs and sophisticated processes were needed. Here, it is demonstrated an electrically tunable $S E R S$ substrate based on tungsten oxides $\left(W O_{3-x}\right)$. Electric field is used to introduce the defects in the oxide on an individual substrate, readily invoking the SERS detection capability, and further tuning the enhancement factor is achieved through electrical programming of the oxide leakage level. Additionally, by virtue of in-situ tuning the defect density and enhancement factor, the substrate can adapt to different molecular concentrations, potentially improving the detection range. These results not only help build a better understanding of the chemical mechanism, they also open an avenue for engaging nonnoble metal materials as multi-functional SERS substrates.
\end{abstract}

KEYWORDS: surface enhanced Raman scattering, transition metal oxide, chemical enhancement, charge transfer, electrical programing, defect density tuning 
The surface-enhanced Raman scattering (SERS) technique has been intensively studied since it was first observed on a roughened silver electrode coated with pyridine in $1973 .{ }^{1}$ It is known for ultra-high sensitivity and specificity when it is used to detect the molecules adsorbed on the $S E R S$ substrate. For this reason, SERS detection has been extensively utilized as a powerful tool for molecular fingerprint tracing in various biological and chemical applications. ${ }^{2-7}$ As the SERS performance is usually determined by material selection, surface morphology of the substrate and the adsorbed analytes, developing novel substrate with attractive enhancement factor $(E F)$, excellent uniformity and reproducibility, additional bio-compatibility and reusability is keenly demanded. $^{8,} 9$ Conventionally, noble metals with optimized surface nanostructures are implemented as the SERS substrates and normally up to 7 8 orders of molecules' Raman scattering signal enhancement can be obtained. ${ }^{10-13}$ The mechanism behind the enhancement observed on noble metal substrates is attributed to the boosted surface electromagnetic field confinement by the surface plasmon, and thus it is termed as the electromagnetic mechanism $(E M)$ for the Raman signal enhancement. ${ }^{14,15}$ However, to make full use of the $E M$, precise control of the metal nanostructures to ensure the strong near-surface electromagnetic field still poses as a significant challenge, especially known for sophisticated experimental design and high costs. In addition, noble metals occasionally show poor stability and biocompatibility when the SERS detection is carried in a harsh environment or biological system, which further limits their applications as the prior-selection analysis platforms. ${ }^{16-19}$

Recently, studies on transition metal oxides $\left(\mathrm{TiO}_{2}, \mathrm{Cu}_{2} \mathrm{O}, \mathrm{MoO}_{3}, \mathrm{ZnO}\right.$ and $\mathrm{WO}_{3}$ etc. $)$, transition metal chalcogenides $\left(\mathrm{Cu}_{2} \mathrm{~S}, \mathrm{Cu}_{2} \mathrm{Se}, \mathrm{MoS}_{2}\right.$ and $\mathrm{WSe}_{2}$ etc. $)$ and two-dimensional materials (graphene, graphene oxide etc.) have demonstrated that non-metal surface could also provide noble-metal comparable SERS enhancement. ${ }^{20-27}$ In these experiments, the Raman signal 
enhancement was mainly ascribed to an increase of charge transfer $(C T)$ between the substrate material and molecules, consequently enhancing the molecule polarizability. Thus, the mechanism is named as the chemical mechanism $(C M)$ for the Raman signal enhancement. ${ }^{28}$ In the meanwhile, a series of elegant papers also have reported that materials like $\mathrm{TiO}_{2}, \mathrm{MoO}_{3}, \mathrm{ZnO}$, $W_{3}$, graphene and $\mathrm{MoS}_{2}$ showed excellent biocompatibility, and some of them were highly resistant to degradation in harsh environment, therefore, the potential use of these materials would greatly expand the application of SERS detection in many fields, e.g. in-vivo health monitoring or chemical reaction tracing. ${ }^{29-34}$ However, essential improvements of the non-metal SERS substrates, such as a simple preparing procedure and achievement of high $E F$, are still demanded.

To-date, established strategies for promoting the CM in SERS lie on the alignment of energy levels between the substrate material and molecules to facilitate the CT. Electrochemical SERS, as a comprehensively discussed technique, has been implemented to manipulate the surface states and promote the $C T$ for $S E R S$ enhancement, however this method requires constant voltage bias and aqueous electrolyte. ${ }^{35,}{ }^{36}$ Recently, defect engineering on the solid-state materials has been extensively used to improve the SERS performance of materials. Compared to the electrochemical method, the defect states in these solid-state electrolytes are nonvolatile, which means that they are able to support the $C T$ even after the removing of the bias. To introduce the defect states in the material, previous reported results have indicated that plasma treatment, ion doping or gas annealing, etc. on materials like $\mathrm{TiO}_{2}, \mathrm{WO}_{3}$ and $\mathrm{ZnO}$ could be effective approaches. ${ }^{22,37-39}$ In the meanwhile, high-energy ultraviolet ( $U V$ ) light was also implemented to create the defects in the substrate oxide. A $U V$-exposed $A u$-nanoparticle embedded oxide substrate showed synergetic enhancement effect; the Raman intensity was raised to a much 
higher level than the case without $U V$ exposure ${ }^{40}$ Furthermore, optimization of chemical synthesis conditions was another extensively adopted approach for adjusting the surface states and achieving significant Raman signal improvement. ${ }^{24,41,42}$ All in all, these experimental practices purposely introduce defects into the material and the SERS enhancement subsequently benefits from the trap-assisted $C T$ between the substrate material and molecules. Nevertheless, they involve a time-consuming process and some of them even require accuracy control of processing conditions. Besides, the defect density as well as the defect level positions in the substrate material is relatively difficult to control through these methods.

Herein, we report the study of the electrically tunable Raman enhancement phenomenon based on the design and fabrication of tungsten oxides $\left(W O_{3-x}\right)$ associated substrate. By electrical programing the defect density in the $W O_{3-x}$ through the oxide leakage current control, the SERS detection capability of the novel substrate can be invoked instantaneously and the enhancement factor can be further precisely modulated. Moreover, the advantage of in-situ electrical defect density control allows the $E F$ of the $S E R S$ substrate that can be tuned and adapted to different molecular concentrations. This gives the SERS substrates a self-adapt capability with potentially improved detection range. The above results not only help affirm the $C M$, they also pave the way towards the implementation of dynamically tunable, self-adaptive non-metal-based SERS substrate for multifunctional and high-sensitivity bio-sensing applications.

\section{Results and Discussion}

$W_{3-x}$ was synthesized by a hydrothermal method. Briefly, tungsten hexachloride $\left(\mathrm{WCl}_{6}\right)$ was used as the tungsten source. It was dissolved in ethanol, and the solution was transferred to a Teflon-lined stainless steel autoclave and kept at $180^{\circ} \mathrm{C}$ for 24 hours. ${ }^{43}$ Thereafter, the obtained blue products $\left(\mathrm{WO}_{3-x}\right)$ were collected by centrifugation, washed with ethanol for several times, 
and finally dried in the air at $60{ }^{\circ} \mathrm{C}$. The morphology of the synthesized $W O_{3-x}$ was detected by scanning electron microscopy $(S E M)$ and transmission electron microscopy (TEM) (see Figure $\mathrm{S} 1$, Supporting Information). From the $S E M$ images, it could be found that the prepared $\mathrm{WO}_{3-x}$ nanoparticle shows multiple-tentacles growing on the core plate. The tentacles entangle with each other, and the lengths of these tentacles vary a lot. In the meanwhile, the TEM image reveals more details. For a typical $W O_{3-x}$ nanoparticle, the size of the core plate is $330 \mathrm{~nm} \times 577$ $\mathrm{nm}$, and high resolution inspection on the single tentacle further evidences that the lattice constant is $0.378 \mathrm{~nm}$, which belongs to (010) planes of monoclinic $\mathrm{WO}_{3}{ }^{37}$ To realize electrical tuning of defect density in the $W O_{3-x}$, the synthesized $W O_{3-x}$ powder was dissolved in ethanol again, sonicated for $15 \mathrm{~min}$ and then spin-coated on a $S i$ wafer with prefabricated 100 -nm-thick $A u$ planar electrodes. After that, the substrate was annealed at $100{ }^{\circ} \mathrm{C}$ for 0.5 hour in the air to improve the adhesion between the $W O_{3-x}$ and the electrodes (see the Methods). Figure 1a and b show the optical image and schematic structure of the prepared SERS substrate. Principally, $W O_{3-x}$ deposited between the gap of the two electrodes constitutes the active $S E R S$ region, where the defect density could be electrically modulated by applying voltage on the two electrodes. Figure 1c shows the atomic force microscopy $(A F M)$ topographical image. It can be seen that the width of the electrodes and the spacing between the two electrodes are both $10 \mu \mathrm{m}$. Figure $1 \mathrm{~d}$ shows the cross-section $S E M$ picture of the $S E R S$ substrate, it reveals that an 80 -nm-thick $W O_{3-x}$ is deposited between the two electrodes during the spin-coating process. In addition, $X R D$ and $U V$-vis spectroscopy were measured for the $W O_{3-x}$ powder sample before and after $100{ }^{\circ} \mathrm{C}$ annealing in the air (Figure S2, Supporting Information). It can be seen from the $X R D$ spectroscopy that both of the pristine and annealed $W_{3-x}$ are monoclinic phases $(P 2 / m, J C P D S$ no. 84-1516). Besides, UV-vis spectroscopy discerns slight blue shift of the absorption curve 
after $100{ }^{\circ} \mathrm{C}$ annealing in the air, indicating the partial removing of deep defects, whereas the weak absorption hump is kept at near-infrared region, which reveals the remaining of shallow defects.

Electrical tuning of the defect density was performed using a Keithley 4200-SCS semiconductor parameter analyzer (see the Methods). A voltage was applied on the left electrode and the right one was always grounded. The average electric field between the electrodes could be evaluated as $\frac{\boldsymbol{V}}{\boldsymbol{d}} \mathrm{V} \cdot \mathrm{cm}^{-1}$, where $\boldsymbol{V}$ is the applied voltage and $\boldsymbol{d}$ is the spacing of the electrodes. Previous understanding revealed that the oxygen vacancy defect density in the $\mathrm{WO}_{3-x}$ layer firmly depended on the leakage current level. ${ }^{44}$ Typically, conductive filaments constituted by oxygen vacancy chains were created during the voltage sweep phase, and the number of the oxygen vacancies in the conductive filaments were determined by the current level sneaking though the oxide; the higher the leakage current, the higher the density. ${ }^{45}$ Therefore, to achieve different defect densities in the oxide, the leakage current level in the oxide are precisely controlled with a preset current compliance $\left(I_{C}\right)$ during the voltage sweep. When the leakage current reaches the compliance value, the voltage sweep is immediately aborted, yielding a defect density that corresponds to the preset $I_{C}$. Figure 2 a shows typical current-voltage $(I-V)$ curves obtained on three similar $S E R S$ substrates with respective preset $I_{C}$ values. The voltage sweeps were carried out in steps. For the first step (when the $W O_{3-x}$ was in the pristine state), a relatively low $I_{C}$ $\left(1 \times 10^{-7} \mathrm{~A}\right)$ was chosen to prevent catastrophic breakdown of the $W O_{3-x}$ when the voltage exceeded a certain threshold voltage $(>100 \mathrm{~V})$. Beyond this voltage, the leakage current rose sharply, signaling a rapid increase of defect generation rate. If this increase was not arrested quickly, a thermal runaway situation that led to a catastrophic breakdown of the $\mathrm{WO}_{3-x}$ would occur. Below the threshold voltage, the leakage current was relatively low $\left(<1 \times 10^{-12} \mathrm{~A} @ 1 \mathrm{~V}\right)$ 
and was comparable for all three substrates. This showed that the $W O_{3-x}$ fabricated by the hydrothermal method was of good quality (low defect density) and uniformity. After the first step, the leakage current of each individual SERS substrate was further programed to the preset $I_{C}$ of $1 \times 10^{-7} \mathrm{~A}, 1 \times 10^{-5} \mathrm{~A}, 1 \times 10^{-3} \mathrm{~A}$, respectively (inset of Figure $2 \mathrm{a}$ ). During the entire two-stage electric field programing periods, the $O$ atom of the weak $W-O$ bond was considered to be outstretched away from its lattice site when the electric field gradually increased in the oxide, leaving the oxygen vacancies behind. ${ }^{46}$ The continuous accumulation of the oxygen vacancies along the oxide ultimately induced the dielectric breakdown, which brought the current to the compliance of $1 \times 10^{-7} \mathrm{~A}, 1 \times 10^{-5} \mathrm{~A}$ and $1 \times 10^{-3} \mathrm{~A}$, respectively.

The SERS performance of the substrates was evaluated by using the Rhodamine $B(R h B)$ as the Raman reporter (the $R h B$ molecular structure is shown in the inset of Figure $2 \mathrm{~b}$ ). However, before collecting the $R h B$ Raman spectra on the substrates, the Raman spectra of the bare $W_{3-x}$ were first discerned (see Figure S3 in Supporting Information). For the $\mathrm{WO}_{3-x}$ powder, Raman peaks that correspond to $O-W-O$ bending and $W-O$ stretching modes are clearly observed, ${ }^{47}$ whereas no evident peak are found on the $80-\mathrm{nm} W O_{3-x}$ film (same thickness as that on the prepared substrate). This discrepancy could be ascribed to fewer number of $O-W-O$ bending and $W-O$ stretching that responses to the laser excitation when the film thickness reduces. Thereafter, in a typical experiment of measuring the $R h B$ Raman spectra, after the successful electrical programing of the defect in the oxide, the bias was removed, and then $10 \mu \mathrm{L}$ of $R h B$ ethanol solution $\left(10^{-4} \mathrm{M}\right)$ was drop-casted on the substrate surface, drying in the atmosphere. The Raman spectra were collected on the $W O_{3-x}$ region between the two Au electrodes under a 532-nm laser excitation (see the Methods). Figure $2 \mathrm{~b}$ shows the Raman spectra with the $\mathrm{WO}_{3-x}$ pre-programed to different leakage current levels. Interestingly, no Raman peaks that related to $R h B$ are detected 
on the pristine $W O_{3-x}$ within the electrodes gap, implying that the chemical interaction between the substrate material and molecules are very weak under defect deficient condition. On the other hand, for the substrate with the leakage current programed to $1 \times 10^{-7} \mathrm{~A}$, it shows that weak Raman signals at 612, 1360, 1507 and $1650 \mathrm{~cm}^{-1}$ (named as P1, P2, P3 and P4) are observed. All of these four Raman peaks are off the $W O_{3-x}$ ones, confirming the Raman enhancement capability of the substrate for the $R h B$ Raman reporters. Thereafter, with the further increase of the preprogramed leakage current to $1 \times 10^{-5} \mathrm{~A}$ and $1 \times 10^{-3} \mathrm{~A}$, it could be found that the intensities of the above Raman peaks are correspondingly increased. Since the defect densities in the oxide are built upon the leakage current level, the increase of Raman intensity with the arising of the leakage current manifests the magnified $C T$ between the substrate material and molecules.

At this stage, it is worth noting that the Raman intensity enhancement of P4 vibrational mode is obviously higher than those of P1, P2 and P3 vibrational modes (Figure S4, Supporting Information). According to earlier studies, this selective enhancement of specific vibrational mode under the $C M$ condition is determined by the nature of the particular atoms or groups that contributes to the vibrational mode. ${ }^{48}$ And for a large molecule like $R h B$, it contains a few vibrational units, thus one vibrational mode always associates with the motions of several vibrational units. In order to uncover the contribution of different vibrational units to a single vibrational mode, previous research works based on density function calculation has been conducted (contribution $<10 \%$ is omitted). ${ }^{49}, 50$ Based on the comprehensive discussion in ref 49 and 50, it can be understood in $R h B$ that $\mathrm{P} 1$ vibrational mode $\left(612 \mathrm{~cm}^{-1}\right)$ comprises two main components, in which $49 \%$ comes from the motion of the xanthene ring and $48 \%$ belongs to the motion of the phenyl ring with the $\mathrm{COOH}$ group. Similarly, for P2 vibrational mode $\left(1360 \mathrm{~cm}^{-1}\right)$, $70 \%$ is contributed by the motion of the xanthene ring and the $\mathrm{NHC}_{2} \mathrm{H}_{6}$ group provides another 
$17 \%$ contributions. For P3 vibrational mode $\left(1507 \mathrm{~cm}^{-1}\right)$, the motion of the xanthene ring produces $69 \%$ contributions and $\mathrm{NHC}_{2} \mathrm{H}_{6}$ group gives another $25 \%$ contributions. Lastly, the motion of the xanthene ring delivers $98 \%$ contributions to the $\mathrm{P} 4$ vibration mode $\left(1650 \mathrm{~cm}^{-1}\right)$. On the other hand, as the $C M$ is intuitively induced by the $C T$ between the molecular orbitals (ground state $\rightarrow$ excited state), and it can be derived from the simulation work indicates that, under the excitation of $532 \mathrm{~nm}$ laser, two-electron transition paths usually happen in the $R h B$. The first major one happens from the highest occupied molecular orbital to the lowest unoccupied xanthene $\pi$ orbital, resulting in the geometry change on the xanthene ring. The second minor one happens from the lowest unoccupied xanthene $\pi$ orbital to an unoccupied phenyl $\pi$ orbital, resulting in the geometry change on the xanthene ring and the phenyl ring. During the Raman measurement, the vibrations of the atoms or groups prefer to mimic the molecular geometries change, consequently bringing in the significant enhanced Raman intensity. Therefore, considering the nature of these four Raman vibrational modes observed on $R h B$, the intensity of $\mathrm{P} 4$ vibrational mode is promoted much more than the other ones. Other than that, the statistical data for the substrates prepared in the same batch are also collected and shown in the Figure S5 of the supporting information. Obviously, substrates with similar leakage levels produce comparable Raman intensities, indicating relatively good performance in uniformity of the SERS intensity. In general, the above observations evidently show the viability of electrically tuning the Raman enhancement through defect density control. Besides, the Raman EFs of the substrate were calculated based on the integrated peak intensity at $1650 \mathrm{~cm}^{-1}$ (details in the Supporting Information), and shown in the Figure 2c. It could be found that the preliminary $E F$ for the substrate with $1 \times 10^{-7} \mathrm{~A}$ leakage current can reach $3.01 \times 10^{5}$. With the further raising of the leakage current levels, the $E F$ can be tuned to higher level, e.g., $3.97 \times 10^{5}$ at $1 \times 10^{-5} \mathrm{~A}$ and 
$1.14 \times 10^{6}$ at $1 \times 10^{-3}$ A. Furthermore, to approximate the lower limit of detection $(L O D)$ for the substrates with $10^{-3}$ A leakage current (substrate with highest $E F$ ) were also evaluated by decreasing the $R h B$ concentration from $10^{-4}$ to $10^{-7} \mathrm{M}$ (see Figure S6 in Supporting Information). Clearly, P1 and P4 peaks are discernible even the molecular concentration reaches $10^{-7} \mathrm{M}$, indicating the superior performance of the programed substrate for detecting low trace analyst. Additionally, the SERS spectra of the other Raman reporters such as Rhodamine $6 G$ (R6G), Crystal Violet (CV) and Methylene Blue (MB) were also collected and shown in Figure S7 of the supporting information. Obviously, the substrate developed in this work also show admirable Raman signal enhancement capability for the above different molecules.

The retention of the $E F$ for the substrate is also evaluated. To achieve this, the programed substrate $\left(10^{-3} \mathrm{~A}\right)$ with $\operatorname{RhB}\left(10^{-4} \mathrm{M}\right)$ was kept in the dry box (atmospheric condition, room temperature and $20 \%$ humidity) for 14 days, and after that, the Raman spectra were collected again. Unfortunately, it could be observed in the Figure $3 \mathrm{a}$ and $\mathrm{b}$ that there are $42 \%$ drop of P1 peak intensity and about $32 \%$ drop of P4 peak intensity, respectively. In an effort to identify the root cause of these evident Raman peak intensity drop, the leakage current of the substrates were measured, and it revealed in the Figure $3 \mathrm{c}$ that it reduced significantly. In other words, large components of the defects in the oxide were eliminated by redoxing reaction during the rest period in the air (Figure S8, Supporting Information), therefore the $C T$ efficient was weakened, resulting in the drop of the Raman peak intensities. However, relying on the flexible electrical tuning ability of the designed SERS substrate based on $W O_{3-x}$ material, it was convenient to reprograming the substrate to the pre-defined current level, as shown in the Figure 3c. The Raman spectra were collected for the refreshed substrate, and the peak intensities (P1 and P4) were extracted as shown in Figure 3a and b again. Apparently, it could be found that P1 and P4 peak 
intensities resume as the leakage current level recovers, which confirms the role of defects in enhancing the Raman signal. At this stage, it is worth noting that the Raman intensity not only can be in-situ recovered to the original intensity through elevating leakage current level, but it also can be electrically tuned down. Nowadays, progress in SERS technology usually can achieve the $L O D$ to $10^{-10} \mathrm{M}$ or even lower. Nevertheless, a high $E F$ may induce signal saturation quickly when the molecule concentration increases, thus restricting the whole detection range, especially for molecules with strong Raman activity. Figure $4 \mathrm{a}$ and $\mathrm{b}$ illustrates the Raman intensity evolution with the lively changing of the leakage current for the designed $W O_{3-x}$-based $S E R S$ substrate. First, the initial leakage current level of the substrate was programed to $1 \times 10^{-3} \mathrm{~A}$ (curve 1, Figure $4 \mathrm{a}$ ) to achieve the maximum $E F$, and then $5 \mu \mathrm{L}$ of $10^{-4} \mathrm{M} R h B$ ethanol solution was dropped on the substrate surface, drying for Raman measurement. As it shows in Figure $4 \mathrm{~b}$ that relatively strong signal intensities of P1 and P4 can be clear observed (curve 1, Figure 4b). In this situation, a negative voltage sweep was applied on the substrate (curve 2, Figure 4a), which reset the leakage current of the substrate to $1 \times 10^{-5}$ A (curve 3, Figure 4 a) due to the partial recovery of the defects. ${ }^{45}$ The Raman spectra were re-measured on this substrate. Since the $E F$ is adequately determined by the defect density, therefore, in the current condition with fewer defects in the oxide, the intensity of P1 and P4 reduces (curve 3, Figure 4b), which is evidently lower than that when the leakage current is $10^{-3} \mathrm{~A}$. The above procedure demonstrates that by readily adjusting the leakage current through the $W O_{3-x}$, the same substrate can be adapted to handle different molecule concentrations, thereby potentially extending the detection range that a single substrate can provide. In addition, it is also confirmed that a positive voltage sweep could set the current back to $1 \times 10^{-3}$ A (curve 4, Figure 4c) and restore the initial high $E F$ (curve 4 , Figure 4b), enabling its capability to detect the low concentration molecules. 
It is well accepted that the Raman enhancement of the semiconductor material is ascribed to the $C M$. The oxygen vacancy, as the main defect morphology in the imperfect semiconductor material, has helped the $C T{ }^{44}$ Herein, to confirm the role of the oxygen vacancy defects for signal enhancement, conductive atomic force microscopy $(C-A F M)$ is used to detect the defect distribution in the $\mathrm{WO}_{3-x}$. Since in the Raman measurement, the penetration depth of the laser in the $W O_{3-x}$ material could be several micrometers, thus the electrons could be readily excited to the conduction band by the injected photons even if the defects are situated below the surface, i.e. within the bulk of the $W O_{3-x}$, however a surface-sensitive technique such as the $C-A F M$ tip is unable to detect these bulk defects, scanning on the untreated surface only gives the extremely low leakage current (Figure S9, Supporting Information). ${ }^{51}$ Therefore, chemical thinning of the $\mathrm{WO}_{3-x}$ was necessary to expose the defect clusters. In this work, $\mathrm{NH}_{3} \cdot \mathrm{H}_{2} \mathrm{O}$ etchant was used to remove a top layer of the $W O_{3-x}$ before the $C$-AFM measurement (see the Methods). ${ }^{52}$ After the chemical thinning of the oxide layer, $C$ - $A F M$ topography and current mapping were carried out with a $10 \mathrm{~V}$ bias applied on the $C-A F M$ tip, and the right $A u$ electrode grounded (inset of Figure $5 b)$. Figure 5a and $\mathrm{c}$ show the 3-dimensional topographical profiles of two substrates, one having a pristine $\mathrm{WO}_{3-x}$ and the other pre-programmed to a leakage current of $1 \times 10^{-3} \mathrm{~A}$, and Figure $5 \mathrm{~b}$ and d show the corresponding current maps. The lateral profile of the SERS substrates, comprising the left and right electrodes and the sandwiched $W O_{3-x}$ (see Figure 1b), are clearly reproduced by the $C-A F M$ topography scan. Additionally, at the right side of the current map of both substrates, a large bright area (corresponding to a saturated leakage current level of $20 \mathrm{nA}$ ) representing the highly conductive right electrode, which distinctly confirms the well-grounded electrodes. In addition, at the left side of the current map, no bright area that represents the electrode could be found for the substrate with $1 \times 10^{-12}$ A leakage current (pristine substrate) on 
Figure $3 \mathrm{~b}$, this is ascribed to the blocked current path between two electrodes in the defect deficient $W O_{3-x}$, and thus no leakage current can be detected on the left electrodes. However, this is not the case for the substrate with $1 \times 10^{-3} \mathrm{~A}$ leakage current, area with saturated current level at the left of the substrate current map still could be clearly observed, indicating the presence of electric field formed conductive path in the oxide (Figure 5d). Furthermore, in the spacing region between the electrodes for the substrate with $1 \times 10^{-12}$ A leakage current, it can be observed that the peak value of the current map is as low as $100 \mathrm{pA}$ only, which is consistent with the electrical measurement. On the other hand, bright shades representing strong leakage paths exposed after chemical thinning could be found on Figure 5d. These high leakage current paths connect the left and right electrodes, comprising the conductive filaments in the sandwiched $\mathrm{WO}_{3-x}$ region, which unambiguously reveals the high density of oxygen vacancy chains in the spacing region.

To further shed light on the mechanism underlying the Raman signal enhancement, firstprinciples simulation was performed. Non-polar $W O_{3-x}$ slabs with oxygen-atom terminated surfaces were used (Figure 6a). A hybrid density functional that based on the semi-local approximation developed by Perdew, Burke and Ernzerhof (PBE) was implemented to correct the underestimated band gap (see the Methods). To investigate the energy level alignment between the $W O_{3-x}$ and $R h B$ molecules with the incorporation of oxygen vacancy, the average electrostatic potential $(A E P)$ was calculated and aligned to the vacuum level which was scaled to $0 \mathrm{~V}$, then the valance band maximum $(V B M)$, conduction band minimum $(C B M)$ and defect energy levels were consequently aligned to the $A E P$ based on the band diagram. ${ }^{53}$ It is shown in Figure $6 \mathrm{~b}$ that the band gap of the $W_{3-x}$ given by the simulation is $\sim 3.5 \mathrm{eV}$, which is consistent with the experimental data and is significantly improved in terms of accuracy as compared to the classical theoretical calculation. ${ }^{54}$ As for the $R h B$ molecules, the highest occupied molecular orbital 
$(H O M O)$ is at $-4.85 \mathrm{eV}$ and the lowest unoccupied molecular orbital (LUMO) is at $-1.64 \mathrm{eV}$. These calculated molecular orbitals also match the reported results. ${ }^{55}, 56$ Since the $\mathrm{WO}_{3-x}$ was terminated with un-passivated oxygen atoms, surface states $(S S)$ were introduced into the band gap even in the slab without oxygen vacancy. However, both of them were mid-gap states and the energy intervals between the neighboring states were still sizable, resulting in inefficient $C T$ under the Raman laser irradiation. On the other hand, the oxygen vacancy brought in an extra level below the $S S$ as evidenced in the band diagram of the $W_{3-x}$ slab with oxygen vacancy. In the meanwhile, the presence of the oxygen vacancy in the oxide also up-shifted slightly the band edges, giving a better alignment between the $C B M$ of $W O_{3-x}$ and the $H O M O$ level of the $R h B$ molecule. ${ }^{57}$ Therefore, it could be expected that those electrons excited under an excitation of $532 \mathrm{~nm}$ were more readily transferred to the molecules with the help of the multi-step defect levels. At this stage, it is worth noting that when more oxygen vacancy defects are accumulated in the oxide, additional defect levels are introduced in the band gap. Because of the lower interlevel energy difference, these supplemental energy levels further facilitate the $C T$, resulting in a greater magnification of the Raman scattering signals.

\section{Conclusion}

In summary, an electrically tunable SERS substrate based on the metal oxide is demonstrated. Experimental investigations have shown that the SERS enhancement factor, from $3.01 \times 10^{5}$ to $1.14 \times 10^{6}$, can be conveniently tuned through the electrical programing of the defect density. Moreover, relying on the advantage of in-situ defect density tuning, the self-adaption capability of the substrate to different molecular concentrations is also evidenced, which provides a facile way to enlarge the detection range. More importantly, theoretical calculations based on firstprinciples are also performed, and the results confirm that the defect levels introduced by the 
oxygen vacancy defects can be well-aligned to the molecular levels, facilitating the $C T$ between the substrate oxide and the molecules. These results not only further enrich the knowledge of the $C M$, they also pave the way towards the implementation of novel, highly flexible non-metalbased SERS substrates with variable detection capability.

\section{Methods}

Materials: $\mathrm{WCl}_{6}(99.99 \%)$ was purchased from Macklin. Ethanol (99.7 \%), $\mathrm{NH}_{3} \cdot \mathrm{H}_{2} \mathrm{O}(25 \%)$, $\mathrm{H}_{2} \mathrm{SO}_{4}(95 \%)$, and $\mathrm{H}_{2} \mathrm{O}_{2}(30 \%)$ were purchased from Sinopharm Chemical Reagent Co., Ltd. All chemicals were used without further purification. In all the experiments, deionized water (resistivity of $18.2 \mathrm{M} \Omega \cdot \mathrm{cm}$ ) was used to prepare the solutions.

Synthesis of $\mathrm{WO}_{3-x^{:}} \mathrm{WO}_{3-x}$ was synthesized by implementing a simple hydrothermal method. Basically, $\mathrm{WCl}_{6}(0.099 \mathrm{~g})$ was used as the tungsten source and dissolved in ethanol $(30 \mathrm{~mL})$, then the prepared solution was transferred to a Teflon-lined stainless-steel autoclave and kept at 180 ${ }^{\circ} \mathrm{C}$ for 24 hours. Thereafter, the obtained blue products were collected by centrifugation (15000 $\mathrm{rpm})$, washed with ethanol for several times, and finally it was dried in the air at $60{ }^{\circ} \mathrm{C}$.

Fabrication of the SERS substrate: The Si wafer was clean by $\mathrm{H}_{2} \mathrm{SO}_{4} / \mathrm{H}_{2} \mathrm{O}_{2}$ solution (3: 1), and then a $100-\mathrm{nm}$ thermal $\mathrm{SiO}_{2}$ was grown in a furnace at $1000{ }^{\circ} \mathrm{C} . \mathrm{Au}(80 \mathrm{~nm}) / \mathrm{Ti}(20 \mathrm{~nm})$ electrodes spaced $10 \mu \mathrm{m}$ apart were fabricated on the $\mathrm{SiO}_{2} / \mathrm{Si}$ substrate by $\mathrm{UV}$ photolithography and electron-beam evaporation of $T i, A u$ metals, and thereafter followed by a lift-off process. To deposit the synthesized $W O_{3-x}$ powder on the pre-prepared substrate, the dried $W O_{3-x}$ powder $(5$ mg) was dissolved in ethanol $(50 \mathrm{~mL})$ again, sonicated for $15 \mathrm{~min}$. Thereafter, $10 \mu \mathrm{L}$ of the prepared solution was drop-casted on the substrate center, and spin-coated onto the substrate at 
$2000 \mathrm{rpm}$ for $30 \mathrm{~s}$. Finally, the substrate was annealed at $100{ }^{\circ} \mathrm{C}$ for 0.5 hour in an argon gas ambient.

Characterization: Voltage sweep and leakage current measurements were performed using a Keithley 4200-SCS semiconductor parameter analyzer system (Tektronix, US). The topographical profile of the substrate and the leakage current map were analyzed by $A F M$ and $C$ $A F M$ on a Cypher S AFM system (Oxford Instruments, UK). For the $C-A F M$ measurement, the substrate was treated with $5 \%$ wt. $\mathrm{NH}_{3} \cdot \mathrm{H}_{2} \mathrm{O}$ at $80{ }^{\circ} \mathrm{C}$ for 20 hours in order to remove the surface $W O_{3-x}$ layer and expose the bulk defects. The $X R D$ spectra were recorded on a D8 Advance diffraction-meter equipped with a LynxEye XE detector (Bruker-AXS, Karlsruhe, Germany). The SEM images of the substrates were acquired by a Hitachi SU-70 system (Hitachi, Japan) under an accelerating voltage of $5 \mathrm{kV}$. The TEM images were obtained on a JEM-2100F transmission electron microscope (JEOL, Japan). The $U V$-vis spectra were collected with a spectrometer (TU1901, P-General, Samutprakarn, Thailand). SERS measurements were made by a Raman microscope equipped with a spectrometer (QE Pro, Ocean Optics, USA). A 532-nm semiconductor laser was used as the excitation source, and the diameter of the spot size of the laser on the substrate is $12.5 \mu \mathrm{m}(100 \times$ objective lens $)$. The Raman spectra were collected under a laser power of $1 \mathrm{~mW}$, and an integration time of $10 \mathrm{~s}$.

First-principles simulation: The simulation work was performed by Vienna Ab-initio Simulation Package $(V A S P) .{ }^{58}$ The ultra-soft pseudo-potential and plane-wave expansions of wave functions and potentials were implemented during the calculation. ${ }^{59}$ The exchange correlation energies were treated within the generalized gradient approximation $(G G A)$ of Perdew, Burke and Ernzerhof $(P B E) .{ }^{60}$ Based on the converging tests, the cut-off energy of 400 $\mathrm{eV}$ for the plane wave basis and k-space grids of $3 \times 3 \times 1$ with the Monkhorst Pack scheme were 
used to calculate the total system energy. For structure optimization, the conjugate gradient method was used and the ion positions were optimized until the residual force was less than 0.01 $\mathrm{eV} \cdot \AA^{-1}$. Moreover, the hybrid density functionals based on the semi-local PBE approximation were implemented by replacing $20 \%$ of $P B E$ exchange with the exact exchange to correct the underestimated band gap. ${ }^{61}$ In the simulation work, an oxygen atom terminated non-polar $\mathrm{WO}_{3}$ slab with 15 - $\AA$ thick vacuum layer was used. The size of the $W O_{3}$ slab is $7.73 \times 7.67 \times 26.67 \AA$, and it contains a total of 12 tungsten atoms and 40 oxygen atoms. To create an oxygen vacancy, one oxygen atom will be removed and the resultant slab was subjected to the same structural optimization procedure. As for the calculating of $R h B$ molecule, an $R h B$ molecule was put in a cell with the size of $30 \times 30 \times 30 \AA$.

\section{AUTHOR INFORMATION}

\section{Corresponding Author}

guchenjie@nbu.edu.cn; zhoujun@nbu.edu.cn; shuwen.zeng@unilim.fr.

\section{ACKNOWLEDGMENT}

C. L. Zhou and L. F. Sun contributed equally to this work. This research was funded by National Natural Science Funding of China (Grant No. 61704095, 61320106014, 61675104), Natural Science Foundation of Zhejiang Province (Grant No. LY19F050002), the "3315" innovative team" Ningbo city, and the K.C. Wong Magna Fund in Ningbo University. This project has also received funding from the European Union's Horizon 2020 research and innovation program under the Marie Sklodowska-Curie grant agreement No. 798916. C. J. Gu 


\section{REFERENCES}

1. Fleischmann, M.; Hendra, P. J.; McQuillan, A. J., Raman Spectra of Pyridine Adsorbed at a Silver Electrode. Chem. Phys. Lett. 1974, 26, 163-166.

2. Yu, B.; Cao, C.; Li, P.; Mao, M.; Xie, Q.; Yang, L., Sensitive and Simple Determination of Zwitterionic Morphine in Human Urine Based on Liquid-Liquid Micro-Extraction Coupled with Surface-Enhanced Raman Spectroscopy. Talanta 2018, 186, 427-432.

3. Xu, J.; Wu, D.; Li, Y.; Xu, J.; Gao, Z.; Song, Y.-Y., Plasmon-Triggered Hot-Spot Excitation on SERS Substrates for Bacterial Inactivation and in Situ Monitoring. ACS Appl. Mater. Interfaces 2018, 10, 25219-25227.

4. $\quad$ Zhou, W.; Tian, Y.-F.; Yin, B.-C.; Ye, B.-C., Simultaneous Surface-Enhanced Raman Spectroscopy Detection of Multiplexed Micro RNA Biomarkers. Anal. Chem. 2017, 89, 61206128.

5. $\quad$ Hu, Y.; Cheng, H.; Zhao, X.; Wu, J.; Muhammad, F.; Lin, S.; He, J.; Zhou, L.; Zhang, C.; Deng, Y.; Wang, P.; Zhou, Z.; Nie, S.; Wei, H., Surface-Enhanced Raman Scattering Active Gold Nanoparticles with Enzyme-Mimicking Activities for Measuring Glucose and Lactate in Living Tissues. ACS Nano 2017, 11, 5558-5566.

6. Lane, L. A.; Qian, X.; Nie, S., SERS Nanoparticles in Medicine: From Label-Free Detection to Spectroscopic Tagging. Chem. Rev. 2015, 115, 10489-10529.

7. Feng, S.; Hu, Y.; Ma, L.; Lu, X., Development of Molecularly Imprinted PolymersSurface-Enhanced Raman Spectroscopy/Colorimetric Dual Sensor for Determination of Chlorpyrifos in Apple Juice. Sens Actuators B: Chem 2017, 241, 750-757.

8. $\quad$ Ding, S.-Y.; Yi, J.; Li, J.-F.; Ren, B.; Wu, D.-Y.; Panneerselvam, R.; Tian, Z.-Q., Nanostructure-Based Plasmon-Enhanced Raman Spectroscopy for Surface Analysis of Materials. Nat. Rev. Mate. 2016, 1, 16021-1-16021-16.

9. Schlücker, S., Surface-Enhanced Raman Spectroscopy: Concepts and Chemical Applications. Angew. Chem., Int. Ed.. 2014, 53, 4756-4795.

10. Jiang, T.; Chen, G.; Tian, X.; Tang, S.; Zhou, J.; Feng, Y.; Chen, H., Construction of Long Narrow Gaps in Ag Nanoplates. J. Am. Chem. Soc. 2018, 140, 15560-15563.

11. Niu, W.; Chua, Y. A. A.; Zhang, W.; Huang, H.; Lu, X., Highly Symmetric Gold Nanostars: Crystallographic Control and Surface-Enhanced Raman Scattering Property. J. Am. Chem. Soc. 2015, 137, 10460-10463. 
12. Liu, K.; Bai, Y.; Zhang, L.; Yang, Z.; Fan, Q.; Zheng, H.; Yin, Y.; Gao, C., Porous AuAg Nanospheres with High-Density and Highly Accessible Hotspots for SERS Analysis. Nano Lett. 2016, 16, 3675-3681.

13. Maiorano, G.; Rizzello, L.; Malvindi, M. A.; Shankar, S. S.; Martiradonna, L.; Falqui, A.; Cingolani, R.; Pompa, P. P., Monodispersed and Size-Controlled Multibranched Gold Nanoparticles with Nanoscale Tuning of Surface Morphology. Nanoscale 2011, 3, 2227-2232.

14. Kneipp, K.; Wang, Y.; Kneipp, H.; Perelman, L. T.; Itzkan, I.; Dasari, R. R.; Feld, M. S., Single Molecule Detection Using Surface-Enhanced Raman Scattering (SERS). Phys. Rev. Lett. 1997, 78, 1667-1670.

15. Xu, H.; Bjerneld, E. J.; Käll, M.; Börjesson, L., Spectroscopy of Single Hemoglobin Molecules by Surface Enhanced Raman Scattering. Phys. Rev. Lett. 1999, 83, 4357-4360.

16. Millo, D.; Bonifacio, A.; Moncelli, M. R.; Sergo, V.; Gooijer, C.; van der Zwan, G., Characterization of Hybrid Bilayer Membranes on Silver Electrodes as Biocompatible SERS Substrates to Study Membrane-Protein Interactions. Colloids Surf. B 2010, 81, 212-216.

17. Runowski, M.; Goderski, S.; Paczesny, J.; Księżopolska-Gocalska, M.; Ekner-Grzyb, A.; Grzyb, T.; Rybka, J. D.; Giersig, M.; Lis, S., Preparation of Biocompatible, LuminescentPlasmonic Core/Shell Nanomaterials Based on Lanthanide and Gold Nanoparticles Exhibiting SERS Effects. J. Phys. Chem. C 2016, 120, 23788-23798.

18. Wang, P.; Wu, Q.; Wang, F.; Zhang, Y.; Tong, L.; Jiang, T.; Gu, C.; Huang, S.; Wang, H.; Bu, S.; Zhou, J., Evaluating Cellular Uptake of Gold Nanoparticles in HL-7702 and HepG2 Cells for Plasmonic Photothermal Therapy. Nanomedicine 2018, 13, 2245-2259.

19. Potara, M.; Boca, S.; Licarete, E.; Damert, A.; Alupei, M.-C.; Chiriac, M. T.; Popescu, O.; Schmidt, U.; Astilean, S., Chitosan-coated Triangular Silver Nanoparticles as a Novel Class of Biocompatible, Highly Sensitive Plasmonic Platforms for Intracellular SERS Sensing and Imaging. Nanoscale 2013, 5, 6013-6022.

20. Sun, L.; Hu, H.; Zhan, D.; Yan, J.; Liu, L.; Teguh, J. S.; Yeow, E. K. L.; Lee, P. S.; Shen, Z., Plasma Modified $\mathrm{MoS}_{2}$ Nanoflakes for Surface Enhanced Raman Scattering. Small 2014, 10, 1090-1095.

21. Jiang, L.; Liang, X.; You, T.; Yin, P.; Wang, H.; Guo, L.; Yang, S., A Sensitive SERS Substrate Based on $\mathrm{Au} / \mathrm{TiO}_{2} / \mathrm{Au}$ Nanosheets. Spectrochim. Acta A 2015, 142, 50-54.

22. Yang, L.; Gong, M.; Jiang, X.; Yin, D.; Qin, X.; Zhao, B.; Ruan, W., Investigation on SERS of Different Phase Structure $\mathrm{TiO}_{2}$ Nanoparticles. J. Raman Spectrosc. 2015, 46, 287-292.

23. Jiang, X.; Song, K.; Li, X.; Yang, M.; Han, X.; Yang, L.; Zhao, B., Double Metal CoDoping of TiO2 Nanoparticles for Improvement of their SERS Activity and Ultrasensitive Detection of Enrofloxacin: Regulation Strategy of Energy Levels. ChemistrySelect. 2017, 2, 3099-3105.

24. Yu, X.; Cai, R.; Song, Y.; Gao, Q.; Pan, N.; Wu, M.; Wang, X., Graphene/TiO ${ }_{2} \mathrm{Hybrid}$ Layer for Simultaneous Detection and Degradation by a One-Step Transfer and Integration Method. RSC Adv. 2017, 7, 14959-14965.

25. Liu, Y.; Gao, Z.; Chen, M.; Tan, Y.; Chen, F., Enhanced Raman Scattering of CuPc Films on Imperfect $\mathrm{WSe}_{2}$ Monolayer Correlated to Exciton and Charge-Transfer Resonances. Adv. Funct. Mater. 2018, 28, 1805710-1-1805710-7.

26. Liu, W.; Bai, H.; Li, X.; Li, W.; Zhai, J.; Li, J.; Xi, G., Improved Surface-Enhanced Raman Spectroscopy Sensitivity on Metallic Tungsten Oxide by the Synergistic Effect of Surface Plasmon Resonance Coupling and Charge Transfer. J. Phys. Chem. Lett. 2018, 9, 40964100 . 
27. Ou, G.; Xu, Y.; Wen, B.; Lin, R.; Ge, B.; Tang, Y.; Liang, Y.; Yang, C.; Huang, K.; Zu, D.; Yu, R.; Chen, W.; Li, J.; Wu, H.; Liu, L.-M.; Li, Y., Tuning Defects in Oxides at Room Temperature by Lithium Reduction. Nat. Comm. 2018, 9, 1302-1-1302-9.

28. Otto, A., The 'Chemical' (Electronic) Contribution to Surface-Enhanced Raman Scattering. J. Raman Spectrosc. 2005, 36, 497-509.

29. Yin, W.; Yan, L.; Yu, J.; Tian, G.; Zhou, L.; Zheng, X.; Zhang, X.; Yong, Y.; Li, J.; Gu, Z.; Zhao, Y., High-Throughput Synthesis of Single-Layer $\mathrm{MoS}_{2}$ Nanosheets as a Near-Infrared Photothermal-Triggered Drug Delivery for Effective Cancer Therapy. ACS Nano 2014, 8, 69226933.

30. Mehrali, M.; Moghaddam, E.; Shirazi, S. F. S.; Baradaran, S.; Mehrali, M.; Latibari, S. T.; Metselaar, H. S. C.; Kadri, N. A.; Zandi, K.; Osman, N. A. A., Synthesis, Mechanical Properties, and in Vitro Biocompatibility with Osteoblasts of Calcium Silicate-Reduced Graphene Oxide Composites. ACS Appl. Mater. Interfaces 2014, 6, 3947-3962.

31. Wen, L.; Chen, L.; Zheng, S.; Zeng, J.; Duan, G.; Wang, Y.; Wang, G.; Chai, Z.; Li, Z.; Gao, M., Ultrasmall Biocompatible $\mathrm{WO}_{3-\mathrm{x}}$ Nanodots for Multi-Modality Imaging and Combined Therapy of Cancers. Adv. Mater. 2016, 28, 5072-5079.

32. Hurst, S. J.; Fry, H. C.; Gosztola, D. J.; Rajh, T., Utilizing Chemical Raman Enhancement: A Route for Metal Oxide Support-Based Biodetection. J. Phys. Chem. C 2011, $115,620-630$.

33. Ji, W.; Zhao, B.; Ozaki, Y., Semiconductor Materials in Analytical Applications of Surface-Enhanced Raman Scattering. J. Raman. Sprctrosc. 2016, 47, 51-58.

34. Zhou, J.; Xu, N. S.; Wang, Z. L., Dissolving Behavior and Stability of ZnO Wires in Biofluids: A Study on Biodegradability and Biocompatibility of $\mathrm{ZnO}$ Nanostructures. Adv. Mater. 2006, 18, 2432-2435.

35. Avila, F.; Ruano, C.; Lopez-Tocon, I.; Arenas, J. F.; Soto, J.; Otero, J. C., How the Electrode Potential Controls the Selection Rules of the Charge Transfer Mechanism of SERS. Chem. Commun. 2011, 47, 4213-4215.

36. Wu, D.-Y.; Li, J.-F.; Ren, B.; Tian, Z.-Q., Electrochemical Surface-Enhanced Raman Spectroscopy of Nanostructures. Chem. Soc. Rev. 2008, 37, 1025-1041.

37. Cong, S.; Yuan, Y.; Chen, Z.; Hou, J.; Yang, M.; Su, Y.; Zhang, Y.; Li, L.; Li, Q.; Geng, F.; Zhao, Z., Noble Metal-Comparable SERS Enhancement from Semiconducting Metal Oxides by Making Oxygen Vacancies. Nat. Comm. 2017, 6, 1038-1-1038-7.

38. Sinha, G.; Depero, L. E.; Alessandri, I., Recyclable SERS Substrates Based on AuCoated ZnO Nanorods. ACS Appl. Mater. Interfaces 2011, 3, 2557-2563.

39. Cao, Y.-Q.; Qin, K.; Zhu, L.; Qian, X.; Zhang, X.-J.; Wu, D.; Li, A.-D., Atomic-LayerDeposition Assisted Formation of Wafer-Scale Double-Layer Metal Nanoparticles with Tunable Nanogap for Surface-Enhanced Raman Scattering. Sci. Rep 2017, 7, 5161-7-5161-8.

40. Ben-Jaber, S.; Peveler, W. J.; Quesada-Cabrera, R.; Cortés, E.; Sotelo-Vazquez, C.; Abdul-Karim, N.; Maier, S. A.; Parkin, I. P., Photo-Induced Enhanced Raman Spectroscopy for Universal Ultra-Trace Detection of Explosives, Pollutants and Biomolecules. Nat. Comm. 2016, 7, 12189-1-12189-5.

41. Yin, Y.; Miao, P.; Zhang, Y.; Han, J.; Zhang, X.; Gong, Y.; Gu, L.; Xu, C.; Yao, T.; Xu, P.; Wang, Y.; Song, B.; Jin, S., Significantly Increased Raman Enhancement on $\operatorname{MoX}_{2}(X=S, S e)$ Monolayers upon Phase Transition. Adv. Funct. Mater. 2017, 27, 1606694-1-1606694-7.

42. Wu, H.; Wang, H.; Li, G., Metal Oxide Semiconductor SERS-Active Substrates by Defect Engineering. Analyst 2017, 142, 326-335. 
43. Tian, Y.; Cong, S.; Su, W.; Chen, H.; Li, Q.; Geng, F.; Zhao, Z., Synergy of $\mathrm{W}_{18} \mathrm{O}_{49}$ and Polyaniline for Smart Supercapacitor Electrode Integrated with Energy Level Indicating Functionality. Nano Lett. 2014, 14, 2150-2156.

44. Trapatseli, M.; Carta, D.; Regoutz, A.; Khiat, A.; Serb, A.; Gupta, I.; Prodromakis, T., Conductive Atomic Force Microscopy Investigation of Switching Thresholds in Titanium Dioxide Thin Films. J. Phys. Chem. C 2015, 119, 11958-11964.

45. Wang, Q.; Puntambekar, A.; Chakrapani, V., Vacancy-Induced SemiconductorInsulator-Metal Transitions in Nonstoichiometric Nickel and Tungsten Oxides. Nano Lett. 2016, $16,7067-7077$.

46. Gu, C.; Zhou, C.; Ang, D. S.; Ju, X.; Gu, R.; Duan, T., The Role of the Disordered $\mathrm{HfO}_{2}$ Network in the High-א n-MOSFET Shallow Electron Trapping. J. Appl. Phys. 2019, 125, 025705-1-025705-7.

47. Sungpanich, J.; Thongtem, T.; Thongtem, S., Large-ScaleSynthesis of $\mathrm{WO}_{3}$ Nanoplates by a Microwave-Hydrothermal Method. Ceram. Int. 2012, 38, 1051-1055.

48. Centeno, S. P.; López-Tocón, I.; Arenas, J. F.; Soto, J.; Otero, J. C., Selection Rules of the Charge Transfer Mechanism of Surface-Enhanced Raman Scattering: The Effect of the Adsorption on the Relative Intensities of Pyrimidine Bonded to Silver Nanoclusters. J. Phys. Chem. B 2006, 110, 14916-14922.

49. Watanabe, H.; Hayazawa, N.; Inouye, Y.; Kawata, S., DFT Vibrational Calculations of Rhodamine 6G Adsorbed on Silver: Analysis of Tip-Enhanced Raman Spectroscopy. J. Phys. Chem. B 2005, 109, 5012-5020.

50. Jensen, L.; Schatz, G. C., Resonance Raman Scattering of Rhodamine 6G as Calculated Using Time-Dependent Density Functional Theory. J. Phys. Chem. A 2006, 110, 5973-5977.

51. Johansson, M. B.; Mattsson, A.; Lindquist, S.-E.; Niklasson, G. A.; Österlund, L., The Importance of Oxygen Vacancies in Nanocrystalline $\mathrm{WO}_{3-\mathrm{x}}$ Thin Films Prepared by DC Magnetron Sputtering for Achieving High Photoelectrochemical Efficiency. J. Phys. Chem. C 2017, 121, 7412-7420.

52. Mutumi, T.; Masaru, K.; Hiroki, O.; Kenji, M., A New Cleaning Technique for X-Ray Masks in Alkaline Solutions by Direct Control of Electrochemical Potential. Jpn. J. Appl. Phys. 2000, 39, 6923-6930.

53. Alkauskas, A.; Broqvist, P.; Pasquarello, A., Defect Energy Levels in Density Functional Calculations: Alignment and Band Gap Problem. Phys. Rev. Lett. 2008, 101 1-4

54. Vasilopoulou, M.; Soultati, A.; Argitis, P.; Stergiopoulos, T.; Davazoglou, D., Fast Recovery of the High Work Function of Tungsten and Molybdenum Oxides via Microwave Exposure for Efficient Organic Photovoltaics. J. Phys. Chem. Lett. 2014, 5, 1871-1879.

55. Zhao, H.; Zhang, Y.; Li, G.; Tian, F.; Tang, H.; Chen, R., Rhodamine B-Sensitized BiOCl Hierarchical Nanostructure for Methyl Orange Photodegradation. RSC Adv. 2016, 6, 7772-7779.

56. Gupta, V. K.; Mergu, N.; Singh, A. K., Rhodamine-Derived Highly Sensitive and Selective Colorimetric and Off-on Optical Chemosensors for Cr3+. Sens Actuators B: Chem 2015, 220, 420-432.

57. Liu, H.; Zeng, F.; Lin, Y.; Wang, G.; Pan, F., Correlation of Oxygen Vacancy Variations to Band Gap Changes in Epitaxial ZnO Thin Films. Appl. Phys. Lett. 2013, 102, 181908-1181908-4.

58. Kresse, G.; Hafner, J., Ab Initio Molecular Dynamics for Liquid Metals. Phys. Rev. B 1993, 47, 558-561. 
59. Vanderbilt, D., Soft Self-Consistent Pseudopotentials in a Generalized Eigenvalue Formalism. Phys. Rev. B 1990, 41, 7892-7895.

60. Perdew, J. P.; Burke, K.; Ernzerhof, M., Generalized Gradient Approximation Made Simple. Phys. Rev. Lett. 1996, 77, 3865-3868.

61. Heyd, J.; Scuseria, G. E.; Ernzerhof, M., Hybrid Functionals Based on a Screened Coulomb Potential. J. Chem. Phys. 2003, 118, 8207-8215. 


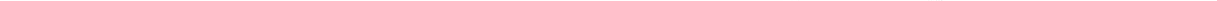


a

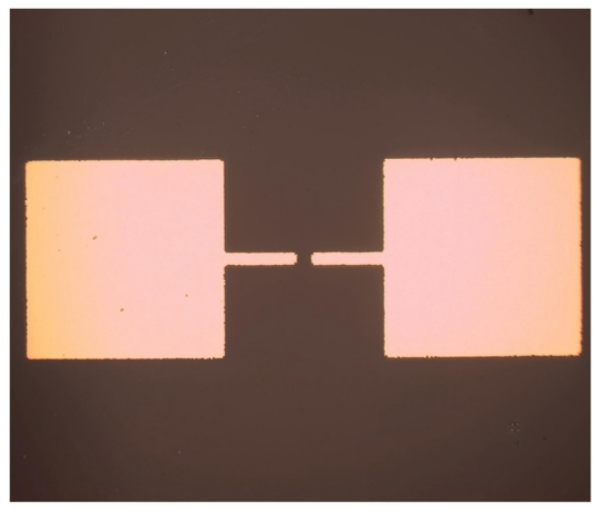

b

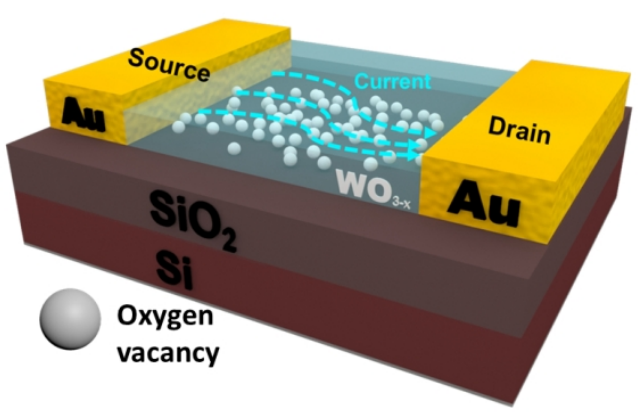

C

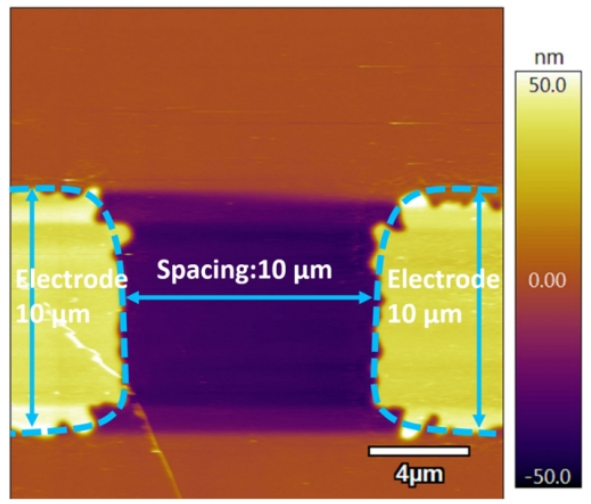

d

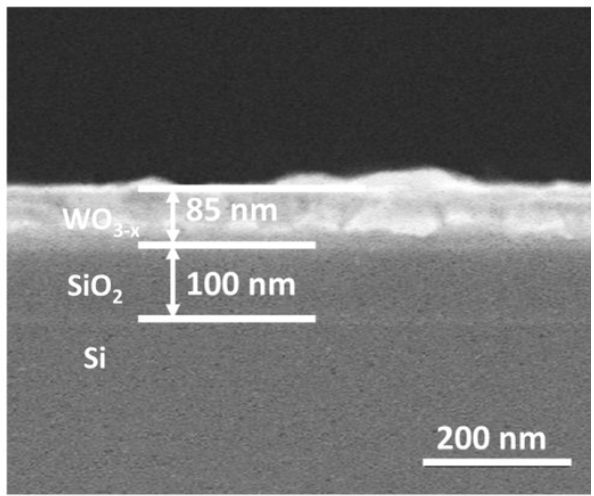

Figure 1. a) Optical image and b) 3D schematic structure of the SERS substrate, c) two-dimensional AFM topographical image, d) cross-section SEM image of the $W_{3-x}$-based SERS substrate.

$557 \times 445 \mathrm{~mm}(72 \times 72 \mathrm{DPI})$ 

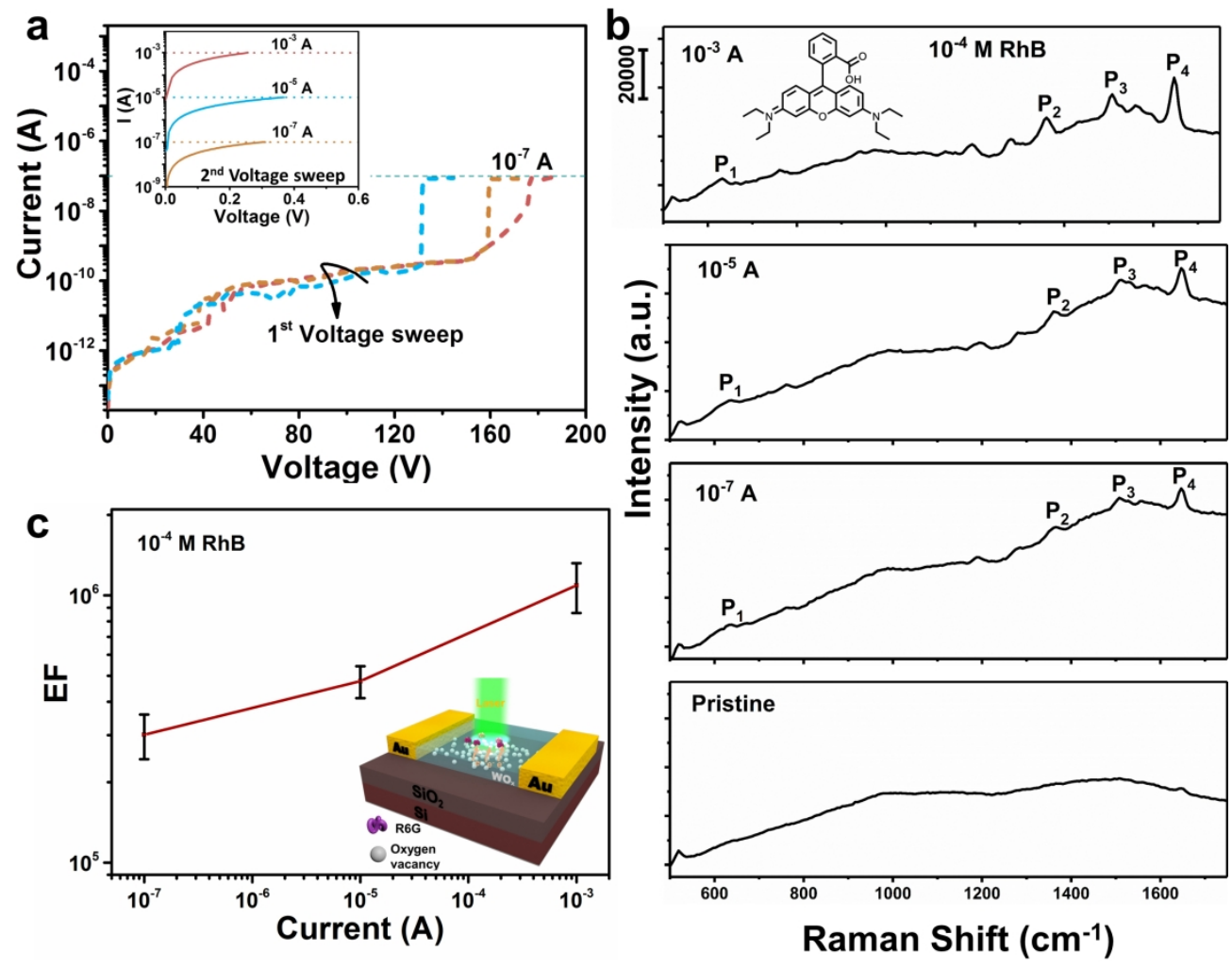

Figure 2. a) Current-voltage (I-V) curves obtained on three similar SERS substrates during the first voltage sweep, b) Raman spectra obtained on a pristine substrate and on the three substrates after programming each of them to a different leakage current, c) SERS enhancement factor as a function of the programmed leakage currents. The inset in (a) shows the second voltage sweep that finally brings the leakage current to the specific compliant current level. The inset in (b) shows the molecular structure of $R h B$.

$916 \times 742 \mathrm{~mm}(72 \times 72 \mathrm{DPI})$ 

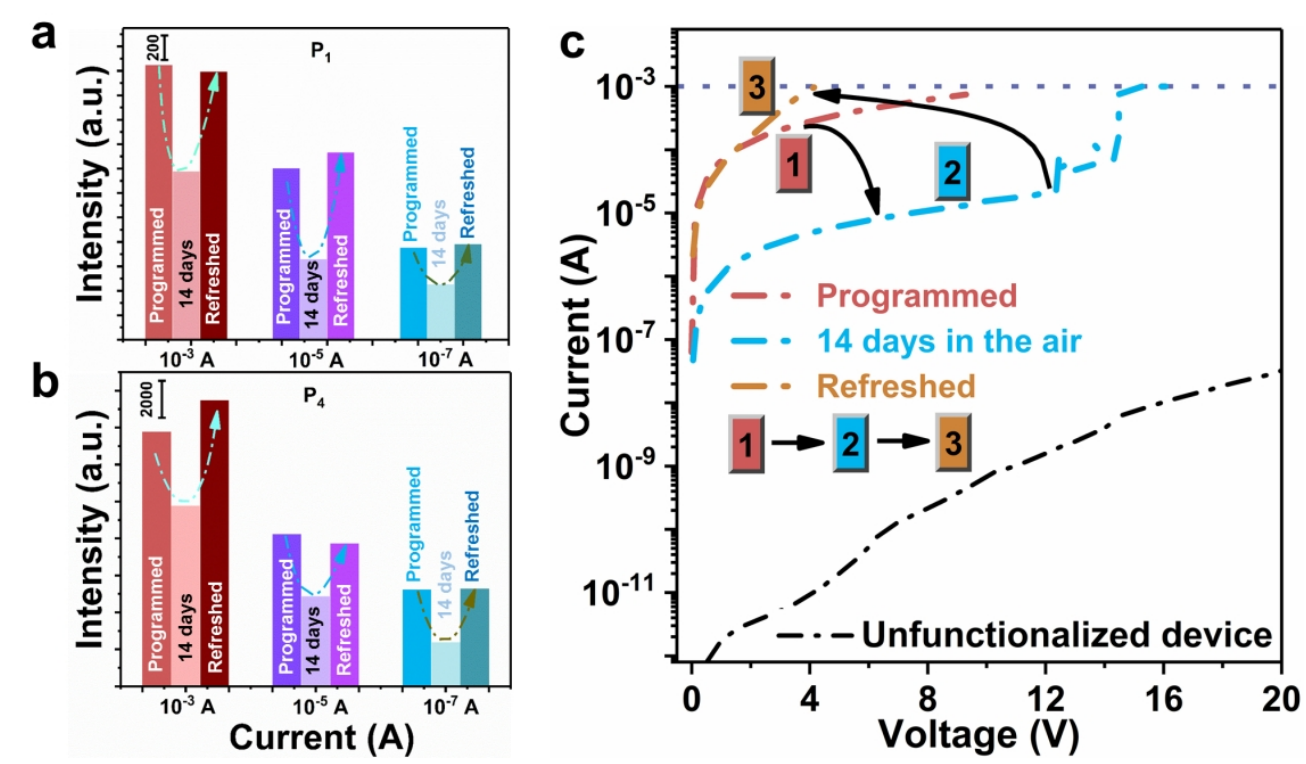

Figure 3. a) The $P 1$ peak intensities measured on the same substrate with three different conditions, $b$ ) the P4 peak intensities measured on the same substrate with three different conditions, $c$ ) the leakage current levels of the substrate that corresponds to the respective peak intensities in (a) and (b).

$707 \times 418 \mathrm{~mm}(72 \times 72 \mathrm{DPI})$ 

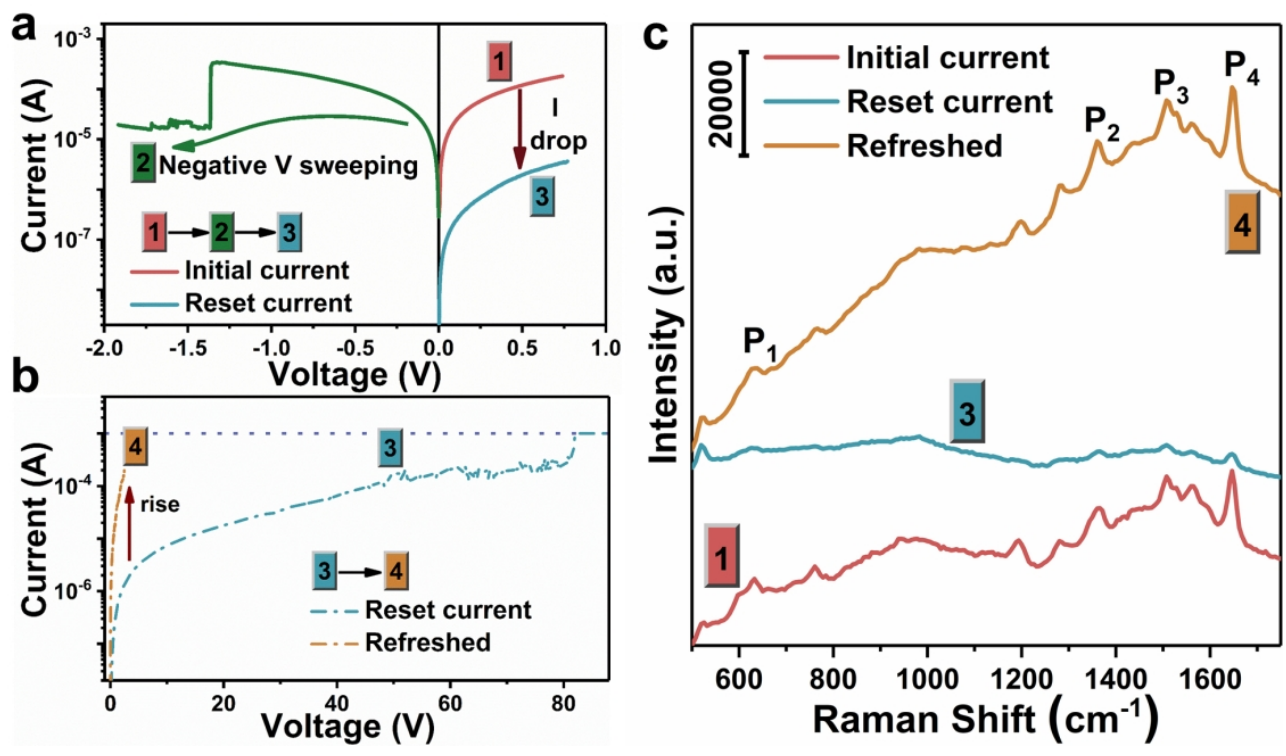

Figure 4. a) Negative voltage sweep that reset the current to lower level. Curve 1 is the initial current level, curve 2 shows the current change when the negative voltage sweep is applied; curve 3 is the current level after negative voltage sweep, b) The evolution of the Raman intensity with in-situ change of the leakage current. The numbers labeled on the respective Raman spectra correspond to the current levels in (a) and $(c), c)$ the leakage current level after reprograming the substrate that has been subjected to the negative voltage sweep.

$698 \times 413 \mathrm{~mm}(72 \times 72 \mathrm{DPI})$ 
a
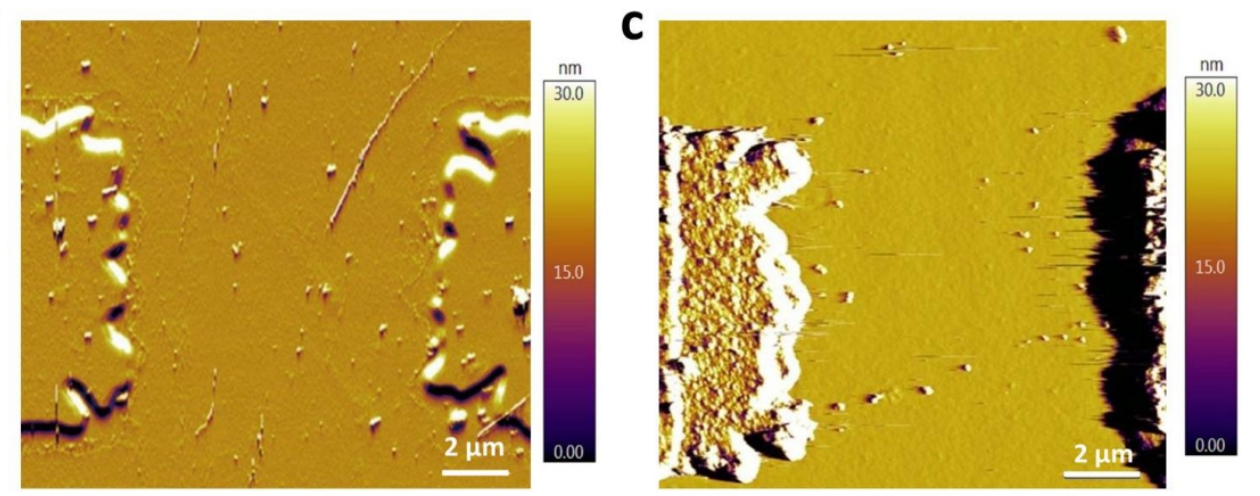

b
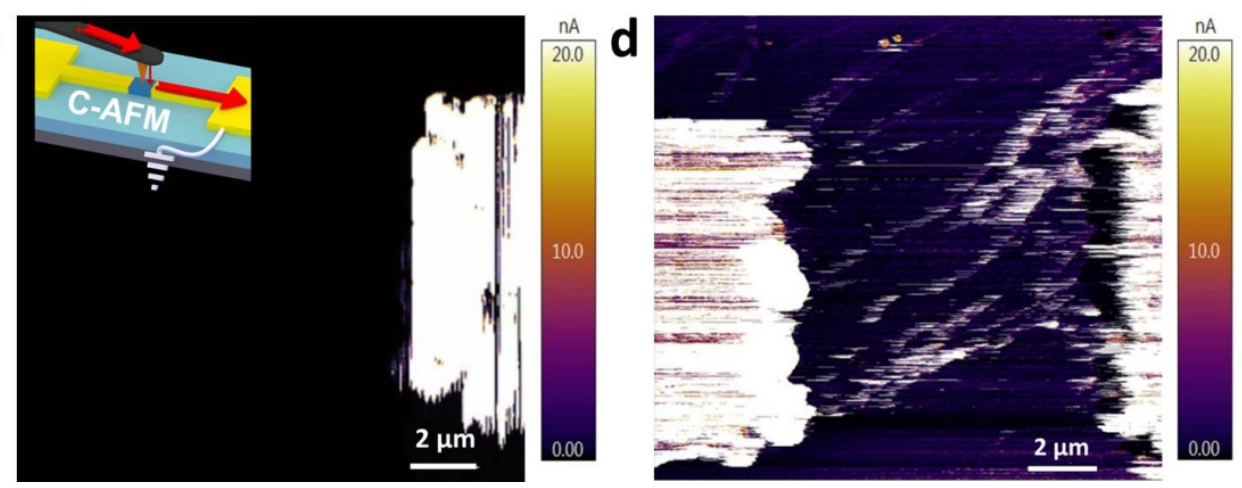

Figure 5. SERS substrate after the chemical thinning. a) The three-dimensional AFM topographical image of a SERS substrate with $1 \times 10^{-12}$ A leakage current, b) the corresponding leakage current map obtained on the same substrate in (a), c) the three-dimensional AFM topographical image of the substrate with $1 \times 10^{-3} \mathrm{~A}$ preprogramed leakage current, d) the corresponding leakage current map obtained on the same substrate in (c). The inset in (b) shows the schematic diagram of the C-AFM measurement. Red arrows show the direction of current flow from the probe towards the grounded right electrode.

$533 \times 412 \mathrm{~mm}(72 \times 72 \mathrm{DPI})$ 

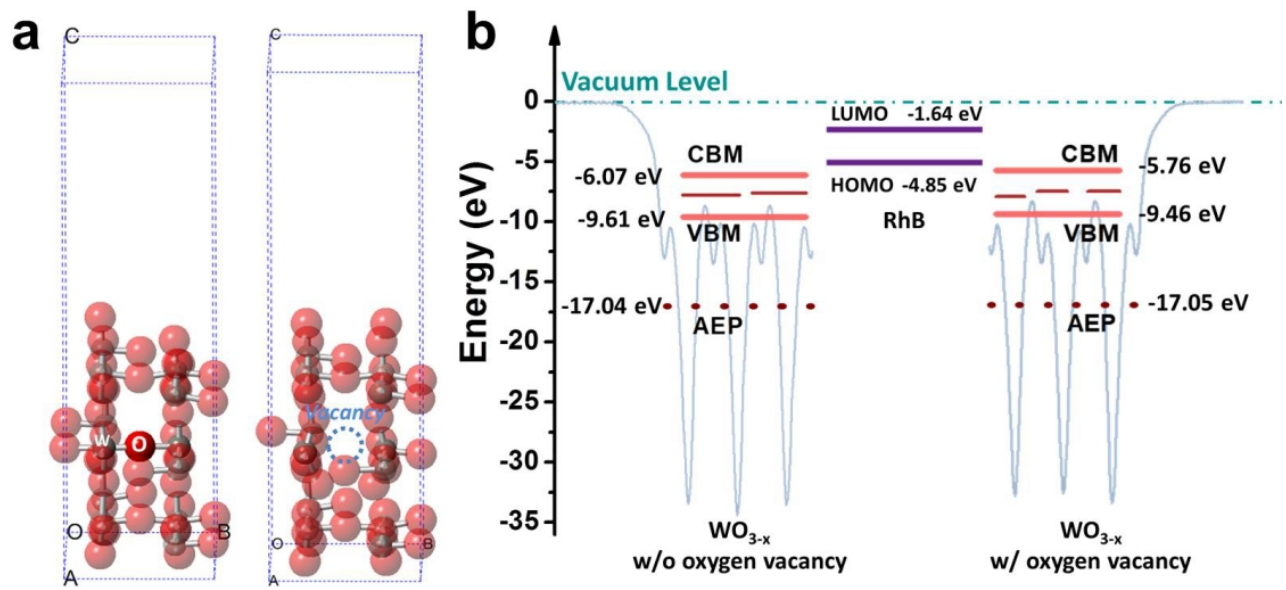

Figure 6. a) Atomic structures of the $\mathrm{WO}_{3-x}$ slabs without oxygen vacancy (left) and with oxygen vacancy (right). In both case, there is a $15-\AA$ thick vacuum layer above the atoms. The large red atoms are oxygen, and the small grey atoms are tungsten, b) alignment of the energy levels of the $R h B$ molecule (center),

$W_{3-x}$ slabs with(left) and without (right) the oxygen vacancy defect. AEP represents the averaged electrostatic potential. CBM means conduction band minimum and VBM means valance band maximum. LUMO represents the lowest unoccupied molecular orbital and HOMO represents the highest occupied molecular orbital.

$573 \times 269 \mathrm{~mm}(72 \times 72 \mathrm{DPI})$ 